\title{
Functional Integration Between the Two Brain Hemispheres: Evidence From the Homotopic Functional Connectivity Under Resting State
}

\author{
Xinhu Jin ${ }^{1 \dagger}$, Xinyu Liang ${ }^{1 \dagger}$ and Gaolang Gong ${ }^{1,2 *}$ \\ 'State Key Laboratory of Cognitive Neuroscience and Learning \& IDG/McGovern Institute for Brain Research, Beijing Normal \\ University, Beijing, China, ${ }^{2}$ Beijing Key Laboratory of Brain Imaging and Connectomics, Beijing Normal University, Beijing, \\ China
}

OPEN ACCESS

Edited by:

Xin Di,

New Jersey Institute of Technology, United States

Reviewed by:

Dapeng Liu,

Johns Hopkins University,

United States

Ye He,

Indiana University, United States

Zhengjia Dai,

Sun Yat-sen University, China

*Correspondence:

Gaolang Gong

gaolang.gong@bnu.edu.cn

tThese authors have contributed equally to this work

Specialty section:

This article was submitted to Brain Imaging Methods, a section of the journal

Frontiers in Neuroscience

Received: 13 June 2020

Accepted: 11 August 2020

Published: 02 October 2020

Citation:

Jin X, Liang X and Gong G (2020)

Functional Integration Between

the Two Brain Hemispheres: Evidence

From the Homotopic Functional

Connectivity Under Resting State.

Front. Neurosci. 14:932.

doi: 10.3389/fnins.2020.00932
Functional integration among neural units is one of the fundamental principles in brain organization that could be examined using resting-state functional connectivity (rsFC). Interhemispheric functional integration plays a critical role in human cognition. Homotopic functional connectivity (HoFC) under resting state provide an avenue to investigate functional integration between the two brain hemispheres, which can improve the present understanding of how interhemispheric interactions affect cognitive processing. In this review, we summarize the progress of HoFC studies under resting state and highlight how these findings have enhanced our understanding of interhemispheric functional organization of the human brain. Future studies are encouraged to address particular methodological issues and to further ascertain behavioral correlates, brain disease's modulation, task influence, and genetic basis of $\mathrm{HoFC}$.

Keywords: functional integration, left and right brain hemispheres, resting-state functional magnetic resonance imaging, resting-state functional connectivity, homotopic functional connectivity

\section{INTRODUCTION}

Brain activity globally interacts at multiple levels with functional integration ranging from neural units to brain regions to overall behavioral output, leading to the emergence of widespread patterns of correlations among neuronal groups (Tononi et al., 1994). How the left and right hemispheres are functionally integrated is a crucial issue in neuroscience. For the two hemispheres, functional integration refers to the interaction between specialized regions, enabling interhemispheric longrange synchronization and information flow (Friston, 2004; van den Heuvel and Sporns, 2013). Such functional integration has been found to play a vital role in cognitive processing, such as visuospatial attention, speech perception and creative thinking (Gotts et al., 2013; Nuttall et al., 2018; Chen et al., 2019).

Various neuroimaging techniques have been applied to investigate functional integration between the two brain hemispheres. For example, Horwitz et al. (1984) used regional metabolic rates acquired by positron emission tomography (PET) to characterize the functional integration among different brain regions. They found that all regions were significantly correlated with their contralateral homologs. Likewise, Duffy et al. (1996) inspected interhemispheric functional integration via resting-state electroencephalogram (EEG) recordings and revealed synchronized 
electrical activity between the left and right hemispheres. With the rapid development of functional magnetic resonance imaging (fMRI) in recent years, resting-state fMRI (rs-fMRI) has been employed to explore interhemispheric functional integration more frequently, given its advantages such as relatively high spatial and temporal resolution without any invasion over other neuroimaging techniques (Biswal et al., 1995). The rsfMRI provides a powerful method to investigate functional integration between different brain regions by calculating restingstate functional connectivity (rs-FC) that is deemed to reflect the interactions across different cerebral regions (Fox and Raichle, 2007). By means of the interhemispheric rs-FC, we could directly quantify functional integration between the two brain hemispheres and thus have a great chance to determine how interhemispheric functional integration affects cognitive processing (Gee et al., 2011).

rs-FC analyses to detect patterns of functional integration between the two brain hemispheres were firstly applied in the motor system (Biswal et al., 1995). Since then, rs-fMRI studies have revealed whole-brain patterns of synchronized spontaneous activity between homotopic regions in left and right hemispheres, which is referred to as homotopic functional connectivity (HoFC) (Salvador et al., 2005; Stark et al., 2008). As demonstrated, HoFC is a robust feature with a peculiar interior distribution corresponding to the functional hierarchy, which does not follow the general law defined by the spatial distance (Salvador et al., 2005; Stark et al., 2008; Tobyne et al., 2016). Meanwhile, it has been found to correlate with the performance of cognitive tasks, such as visuospatial attention (Gracia-Tabuenca et al., 2018) and executive function (Zhao et al., 2020). As summarized in Table 1, a number of studies have achieved progress in functional integration between the two brain hemispheres by virtue of HoFC measurements under resting state.

This review aims to summarize the studies of functional integration between the two brain hemispheres by using HoFC under resting state and to highlight how the findings have enhanced our understanding of hemispheric functional organization in the human brain. For the summary of existing literatures, we included six sections: (1) Methods of Calculating HoFC, (2) Physiological Effects on HoFC, (3) Structural Foundation of HoFC, (4) Correlation Between HoFC and Cognition, (5) HoFC Abnormalities in Brain Diseases, and (6) the Role of HoFC in Functional Lateralization. Future considerations are elaborated into four topics: (1) Lifespan Trajectory of HoFC, (2) Genetic Basis of HoFC, (3) HoFC Under Tasking State, and (4) the Functional Meaning of HoFC to Cognition in Healthy People.

\section{HOFC AND INTERHEMISPHERIC FUNCTIONAL INTEGRATION}

In this part, we review relevant rs-fMRI findings of functional integration between the two brain hemispheres by using HoFC.

\section{Methods of Calculating HoFC}

As shown in Figure 1, HoFC is calculated by Pearson's correlation between the time series of homotopic locations under resting state, which is the most important way to reflect functional integration between the two brain hemispheres. In early stages, homotopic locations are defined as corresponding anatomical areas or spatial locations in opposite hemispheres (Salvador et al., 2005; Stark et al., 2008; Zuo et al., 2010). Recently, researchers proposed that each brain region has a unique homotopic contralateral counterpart with a maximal rs-FC (Joliot et al., 2015). Specifically, HoFC is computed by using different methodological definitions with various spatial scales, such as regions of interest (ROIs), voxel-level in volume, or vertex-level on cortical surface. Below, we summarized different methods to obtain HoFC.

\section{Atlas-Based HoFC}

The majority of early studies adopted anatomical parcellations to define homotopic locations in the left and right hemispheres. Initially, rs-fMRI studies mostly concentrated on a specific cortical or subcortical brain region and have observed a high level of interhemispheric correlation in those regions (Biswal et al., 1995; Lowe et al., 1998; Cordes et al., 2000). Until 2005, Salvador et al. (2005) first noticed that synchronous activity across homotopic regions was a ubiquitous phenomenon among the whole brain. This first whole-brain analysis based on human rs-fMRI data calculated the HoFC via the automated anatomical labelling (AAL) atlas (Salvador et al., 2005), which is proposed by Tzourio-Mazoyer et al. (2002) and comprises 45 corresponding anatomical ROIs in each cerebral hemisphere. Based on AAL atlas as well, Shen et al. (2015) built on their work under resting state and found HoFC was significantly higher than heterotopic or intrahemispheric FC, validated in both human and macaque datasets (Shen et al., 2015). In addition, they discovered HoFC was more stable and resistant across both varied conditions and temporal changes than heterotopic or intrahemispheric FC (Figure 2A). In 2008, Stark et al. (2008) used the HarvardOxford Structural Atlas to divide the entire cerebrum into 112 homotopic regions, 56 in each brain hemisphere. They reported a pattern of regional variation in temporally correlated lowfrequency brain activity between the two hemispheres, suggesting that interhemispheric coordination may vary across homotopic regions in the human brain. As shown in Figure $\mathbf{2 B}$, despite interhemispheric HoFC across the whole brain regions was robust, higher HoFC value was more exhibited in primary sensorimotor cortices than in higher-order association areas. In recent years, studies have suggested that it would be more reasonable and advantageous for the investigation of cerebral functional organization by using homotopic ROIs derived from functional parcellation (Joliot et al., 2015; Tzourio-Mazoyer et al., 2016). Therefore, Joliot et al. (2015) developed a functional parcellation based on the intrinsic connectivity of homotopic areas, namely the Atlas of Intrinsic Connectivity of Homotopic Areas (AICHA). This atlas covers the entire cerebral gray matter and includes 192 homotopic region pairs, which has already been used in studies related to HoFC (Tzourio-Mazoyer et al., 2016).

\section{Voxel-Based HoFC}

In order to investigate the HoFC on a finer scale, Zuo et al. (2010) provided a comprehensive examination of HoFC by 
TABLE 1 | Basic information for published rs-fMRI studies of HoFC.

\begin{tabular}{|c|c|c|c|c|}
\hline Author (years) & Modeling method & Scale & Scope & Main findings \\
\hline Quigley et al. (2003) & Volume-based & Voxel-level & Seed regions & C \\
\hline Salvador et al. (2005) & Volume-based & ROI-level & Whole brain & A \\
\hline Stark et al. (2008) & Volume-based & ROI-level & Whole brain & A \\
\hline Johnston et al. (2008) & Volume-based & ROI-level & Seed regions & C \\
\hline Zuo et al. (2010) & Volume-based & Voxel-level & Whole brain & $\mathrm{A} / \mathrm{B}$ \\
\hline Smyser et al. (2010) & Volume-based & ROI-level & Seed regions & B \\
\hline Kelly et al. (2011) & Volume-based & Voxel-level & Whole brain & $E$ \\
\hline Hoptman et al. (2012) & Volume-based & Voxel-level & Whole brain & $E$ \\
\hline Owen et al. (2013) & Volume-based & ROI-level & Seed regions & C \\
\hline Guo et al. (2013) & Volume-based & Voxel-level & Whole brain & $E$ \\
\hline Yang et al. (2014) & Volume-based & Voxel-level & Whole brain & $E$ \\
\hline Shen et al. (2015) & Volume-based & ROI-level & Whole brain & $A$ \\
\hline Tzourio-Mazoyer et al. (2016) & Volume-based & ROI-level & Whole brain & $\mathrm{B} / \mathrm{F}$ \\
\hline Ren et al. (2015) & Volume-based & Voxel-level & Whole brain & $E$ \\
\hline Luo et al. (2015) & Volume-based & Voxel-level & Whole brain & $E$ \\
\hline Tobyne et al. (2016) & Surface-based & Vertex-level & Whole brain & A \\
\hline Emerson et al. (2016) & Volume-based & ROI-level & Seed regions & $\mathrm{B}$ \\
\hline Hermesdorf et al. (2016) & Volume-based & Voxel-level & Whole brain & $E$ \\
\hline Roland et al. (2017) & Volume-based & ROI-level/voxel-level & Seed regions/whole brain & C \\
\hline Guo et al. (2018) & Volume-based & Voxel-level & Whole brain & $E$ \\
\hline Gracia-Tabuenca et al. (2018) & Volume-based & Voxel-level & Whole brain & $\mathrm{B} / \mathrm{D} / \mathrm{F}$ \\
\hline Mollink et al. (2019) & Volume-based & ROI-level & Whole brain & C \\
\hline Fan et al. (2018) & Volume-based & Voxel-level & Whole brain & $E$ \\
\hline Luo et al. (2018) & Volume-based & Voxel-level & Whole brain & $E$ \\
\hline Zhao et al. (2020) & Volume-based & Voxel-level & Whole brain & $B / D$ \\
\hline Chen et al. (2020) & Volume-based & Voxel-level & Whole brain & E \\
\hline Ye et al. (2020) & Volume-based & Voxel-level & Whole brain & $E$ \\
\hline
\end{tabular}

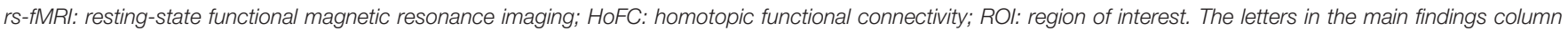

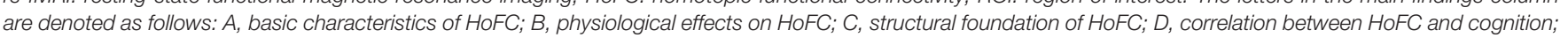
$E$, HoFC abnormalities in brain diseases; $F$, the role of $\mathrm{HoFC}$ in functional lateralization.

using a voxel-wise measure called voxel-mirrored homotopic connectivity (VMHC). Specifically, the individual rs-fMRI data were registered to a symmetrical brain template in Montreal Neurological Institute (MNI) space, which was generated by averaging the group-specific mean normalized T1 image and its left-right mirrored image. This registration step roughly ensures each voxel in one hemisphere has a homotopic voxel in contralateral hemisphere. Then, the Pearson's correlation coefficient between the resting-state time series of each voxel and its symmetrical interhemispheric counterpart was calculated as the VMHC. By using this method, they also verified consistent regional variation of HoFC with Stark et al. (2008). In contrast to the atlas-based HoFC, the VMHC approach allows a greater and finer appreciation of regional variation within larger cortical structures, such as the cingulate, precentral and postcentral gyri (Zuo et al., 2010).

\section{Surface-Based HoFC}

To achieve a better alignment of homologous location between the two brain hemispheres, a surface-based HoFC can be used (Jo et al., 2012).

Using the landmark-based anatomical correspondence, Jo et al. (2012) first putatively established vertex-wise homotopic

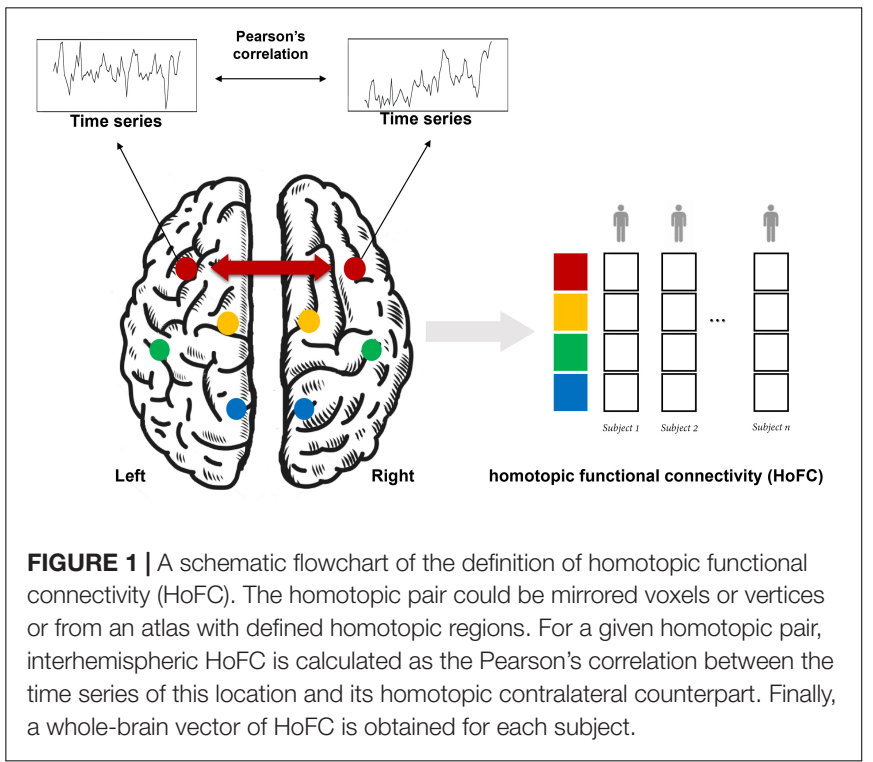

locations between the two hemispheres on a non-symmetric template brain. They identified the improved precision of this 
A

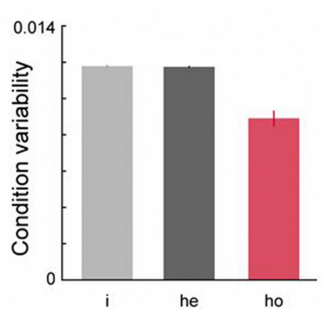

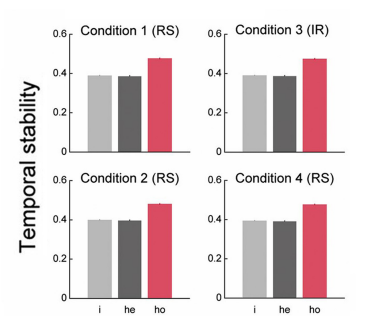

B

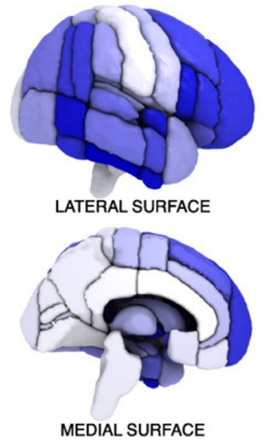

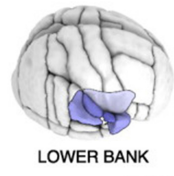
SYLVIAN FISSURE

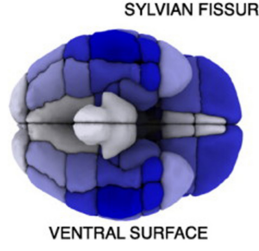

C

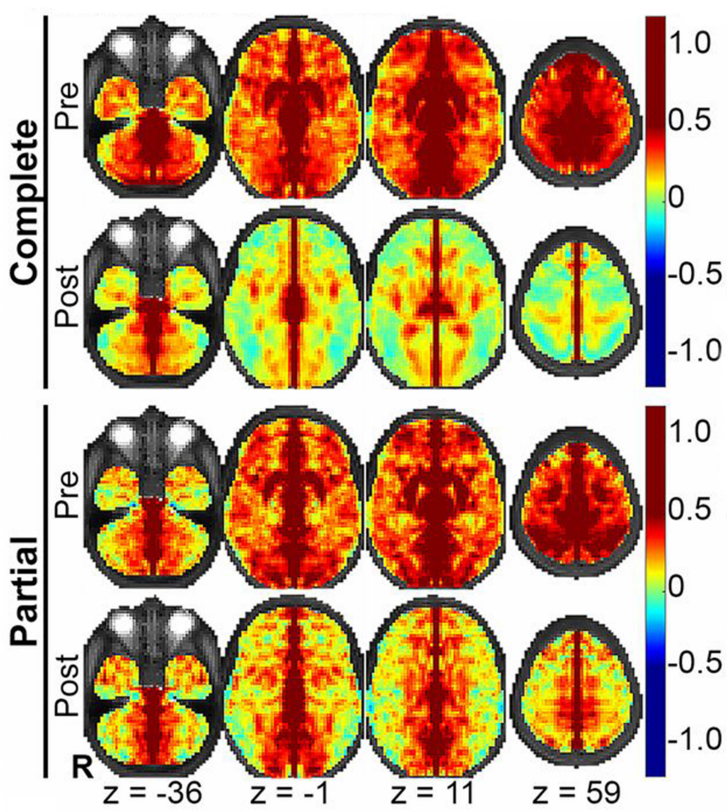

FIGURE 2 | The basic characteristics and structural foundation of HoFC. (A) The condition variability and temporal stability for different types of functional connectivity, including intrahemispheric (i), heterotopic (he), and homotopic (ho). The figure is adapted from Shen et al. (2015). Permission to reuse Figures 2, 3 from the article was granted by PNAS. (B) Mean interhemispheric HoFC indicated for all cortical regions. The figure is adapted from Stark et al. (2008). Permission to reuse Figure 4 from the article was granted by Society for Neuroscience ("Copyright [2008] Society for Neuroscience"). (C) HoFC computed as the Fisher Z-transformed VMHC before (first and third rows) and after (second and forth rows) corpus callosotomy (complete and partial). The figure is adapted from Roland et al. (2017). Permission to reuse Figure 4 from the article was granted by PNAS.

surface-based approach over the previous volume-based flipping method. Following this study, Tobyne et al. (2016) further introduced a computationally efficient technique, surface-based HoFC, that leveraged surface-based registration to improve the spatial specificity and accuracy of cortical HoFC under resting state. The surface-based HoFC for each pair of homologous vertices was calculated by using the pairwise Pearson's correlation between the extracted time series. This technique is presented as a sensitive measure of HoFC in the human brain and is proved to be reliable both within and across subjects (Tobyne et al., 2016). Particularly, this method presents a more constrained pattern of HoFC along the medial surface. As noted, either the volumetric smoothing or greater accuracy of spherical registration techniques might lead to discrepancies between volume-based and surface-based HoFC (Tobyne et al., 2016).

\section{Physiological Effects on HoFC}

The human brain is affected by a number of physiological factors, such as age and sex. Zuo et al. (2010) systematically investigated age-related $\mathrm{HoFC}$ changes under resting state by using VMHC in 214 healthy individuals (age range: 7-85 years). As shown in Figure $\mathbf{3 A}$, sensorimotor regions displayed a HoFC increase, whereas higher-order association regions showed a HoFC decrease along with age. In addition, they detected complex maturational curves, such as the quadratic trajectories of insula and lingual gyrus as well as the cubic trajectories of putamen and superior frontal gyrus. Furthermore, sex-related differences in HoFC between males and females were discovered, with males having weaker HoFC in the medial and dorsal regions as well as greater $\mathrm{HoFC}$ in the parahippocampal and fusiform gyri (Figure 3B). Moreover, there were interactions between age and sex on resting-state $\mathrm{HoFC}$ in the dorsolateral prefrontal cortex and amygdala. In a recent study, Zhao et al. (2020) examined VMHC in 93 healthy volunteers between 19 and 85 years of age and observed reductions in VMHC of the ventromedial prefrontal cortex, inferior parietal lobule, dorsal anterior cingulate cortex, hippocampus and insula along with age. In addition to these VMHC approaches, TzourioMazoyer et al. (2016) used the functionally driven homotopic atlas (AICHA) to define the homotopic regions of the human brain. They recruited 291 individuals (age range: 18-57 years; 148 men, 143 women) and found that mean HoFC (averaged across the 36 pairs of lateral homotopic regions) significantly decreased with age and was larger in males than females. Together, HoFC in lifespan indicated a global decrease related with age (Zuo et al., 2010; Tzourio-Mazoyer et al., 2016). In specific regions, Zhao's study found negative correlation, while Zuo's study reported various development trajectories (Zuo et al., 2010; Zhao et al., 2020). The discrepancies might be due to different statistical models in their studies, with a simple GLM in Zhao's work and a more complicated one including both quadratic and cubic terms in Zuo's work. In Zuo et al. (2010), the age distribution is skewed, which could affect the results of relationship between age and HoFC. Unlike the three 

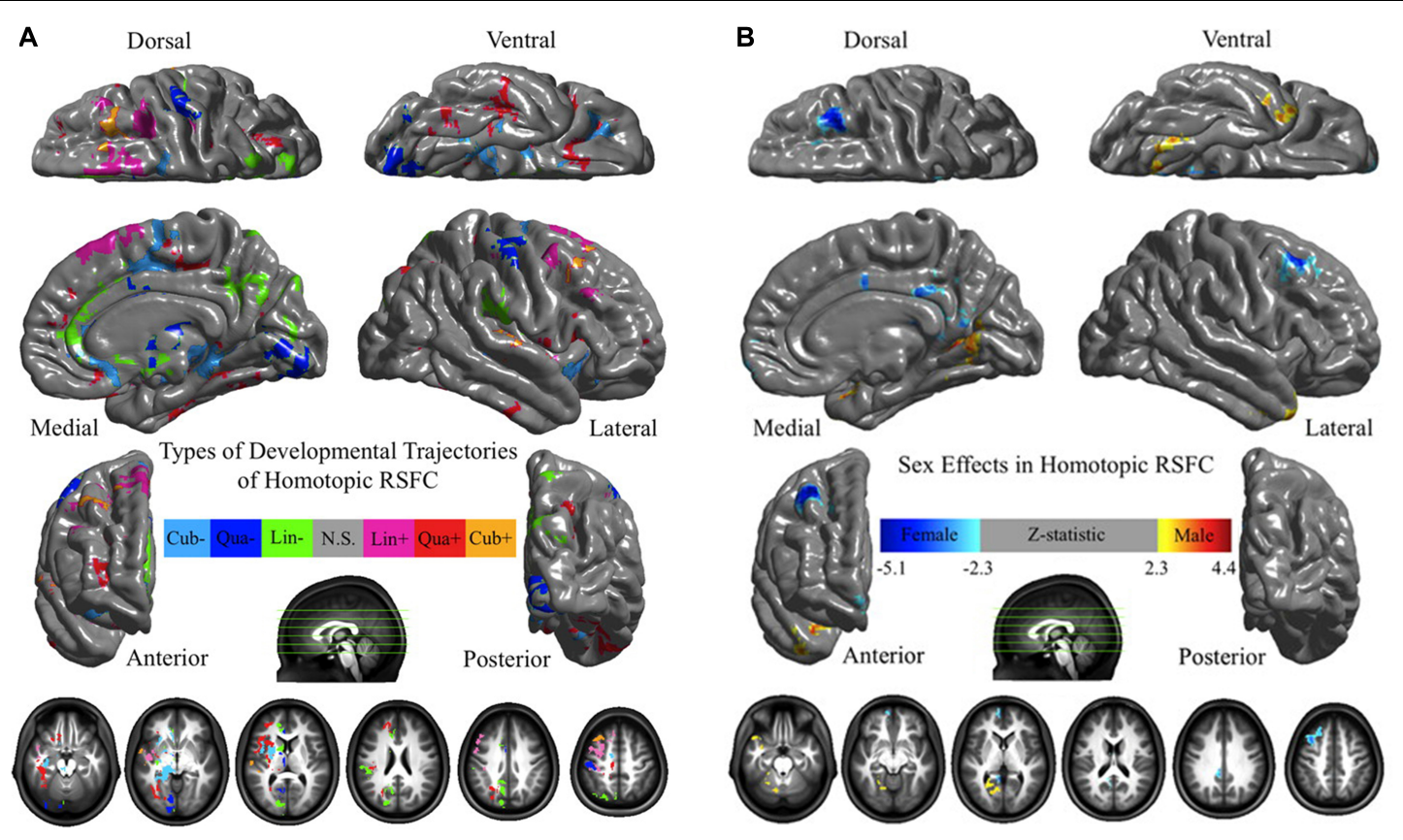

FIGURE 3 | The physiological effects on HoFC. (A) Age effects on HoFC. The linear (Lin), quadratic (Qua), and cubic (Cub) effects of age on VMHC were shown at each voxel, evaluating through multiple linear regressions model. (B) Sex effects of HoFC. One-sample tests on regression coefficients of sex was performed for both positive and negative contrasts. These figures are adapted from Zuo et al. (2010). Permission to reuse Figures $\mathbf{3}, \mathbf{5}$ from the article was granted by the Creative Commons Attribution-Non-commercial-Share Alike 3.0 Unported License (CC-BY-NC-SA).

studies above, Gracia-Tabuenca et al. (2018) focused on typically developing children ranging in age from 6 to 10 years. In a sample of 60 participants, significant sex effects were noted in the frontal pole, but no age effects were identified.

In infant group, Emerson et al. (2016) tried to portray the development curve of interhemispheric functional asymmetry during the first 2 years of life, by leveraging a large sample of human infants with longitudinal rs-fMRI scans. The interhemispheric HoFC exhibited three predominant patterns, including one trajectory for sensory regions, one trajectory for higher-order association regions and another trajectory for language-related regions. Concentrated on an earlier stages from 26 weeks postmenstrual age (PMA), Smyser et al. (2010) obtained resting-state networks based on rs-FC data of preterm infants, and they found the networks with more mature forms in older infants consisted of localized interhemispheric HoFC between homotopic counterparts, demonstrating a regionally age-specific development pattern.

\section{Structural Foundation of HoFC}

Like other rs-FC, HoFC is likely constrained by structural connections of the brain (Honey et al., 2009). Anatomically, the corpus callosum (CC) is the major neural pathway that connects the homotopic regions of the left and right hemispheres (Innocenti, 1986) and putatively plays a pivotal role in mediating HoFC under resting state (Tzourio-Mazoyer et al., 2016; Gracia-Tabuenca et al., 2018).

This line of research was explored mainly by using patients with CC defects. In 2003, Quigley et al. (2003) found that patients with agenesis of the corpus callosum (AgCC) showed diminished interhemispheric functional connectivity in the motor and auditory cortices compared with healthy participants. Owen et al. (2013) extracted 17 resting-state networks of patients with AgCC (including partial and complete) and well-matched healthy controls. They detected a striking loss of interhemispheric HoFC of the insularopercular regions, posterior cingulate cortex, and precuneus in AgCC subjects. Johnston et al. (2008) presented a rs-FC study of a child patient with medically refractory epilepsy both before and after complete section of the CC, which demonstrated reduced interhemispheric HoFC and preserved intrahemispheric connectivity postoperatively. Further, Roland et al. (2017) evaluated rs-FC in 22 patients with intractable epilepsy after surgical section of the CC; they found markedly reduced interhemispheric $\mathrm{HoFC}$ in the human brain, which was more profound in multimodal associative areas than in primary regions. The existence of polysynaptic FC between distant cerebral regions was inferred by comparison of partial vs. complete callosotomy (Figure 2C). These results together indicated that callosal anatomical connections played a critical role in the existence and maintenance of interhemispheric HoFC, which provided crucial insights into general relationship between structural connection and functional connectivity in the human brain. These approaches, however, were unable to characterize interindividual differences of healthy people because of the particularity of subjects with CC defects, although they did have relatively high biological specificity and interpretability. 
A large number of studies have applied diffusion magnetic resonance imaging (dMRI) to study structural connections of the human brain non-invasively. Using dMRI and rs-fMRI data in 11354 subjects from the UK Biobank, Mollink et al. (2019) focused on interhemispheric connectivity and corresponding commissural fibers passing through the CC between pairs of homotopic cerebral regions. They constructed multivariate models to link interhemispheric structural connectivity and functional connectivity, showing that ninety percent of human brain regions were statistically related between HoFC under resting state and white matter microstructure of CC.

In summary, the results both in patients and healthy people provide strong evidence supporting that corticocortical CC connectivity serves as pivotal structural basis underlying the resting-state HoFC between homotopic cortical regions of the two hemispheres.

\section{Correlation Between HoFC and Cognition}

As an important representation of functional integration between the two brain hemispheres, HoFC under resting state was found to be significantly correlated with human's cognitive activities, such as visuospatial attention and executive function. GraciaTabuenca et al. (2018) discovered that the VMHC of the occipital fusiform gyrus and the lingual gyrus was negatively associated with the performance in visual long-term memory tasks. In addition, Zhao's study found that the VMHC of the ventromedial prefrontal cortex and insula showed a negative linear relationship with the performance on the color-word inhibition task mainly about executive function (Zhao et al., 2020).

\section{HoFC Abnormalities in Brain Diseases}

A number of studies have made direct comparisons of HoFC between different patient groups and healthy controls, most of which utilized VMHC to represent HoFC.

Compared to healthy controls, some studies have demonstrated increased HoFC in patient groups, such as idiopathic generalized epilepsy (Yang et al., 2014) and paroxysmal kinesigenic dyskinesia (Ren et al., 2015), while others reported decreased HoFC, ranging from cocaine addiction (Kelly et al., 2011), schizophrenia (Hoptman et al., 2012), Parkinson's disease (Luo et al., 2015), major depressive disorder (MDD) (Hermesdorf et al., 2016), to amnestic mild cognitive impairment (Luo et al., 2018). Some brain diseases, including HBV-related cirrhosis (Ye et al., 2020) and subacute stroke (Chen et al., 2020), showed both increases and decreases of HoFC in different brain regions. Although majority of results are consistent across studies, discrepancies exist. For example, several studies have used VMHC under resting state to explore the difference between MDD patients and healthy people (Guo et al., 2013, 2018; Hermesdorf et al., 2016; Fan et al., 2018). Compared to healthy controls, most studies found no VMHC increase and a significant VMHC decrease in the cuneus, posterior cingulate cortex and precuneus in MDD patients. However, Hermesdorf et al. (2016) reported a VMHC decrease in the putamen, superior temporal gyrus and insula, which exhibited a wider brain region change in the VMHC in patients with MDD than other studies. These discrepancies might be due to the differences among studies in inclusion criteria and the number of participants, fMRI acquisition, analysis methods, and so on. Regarding the relationship with behavior and symptoms, the HoFC under resting state in specific brain regions was found to be associated with scores of disease-related scales in patient groups. For instance, Kelly et al. (2011) revealed a correlation between HoFC within the regions in the dorsal attention network and self-reported lapses of attention in the cocaine-dependent group. For schizophrenia, Hoptman et al. (2012) showed that HoFC in the postcentral gyrus extending into the precentral gyrus was associated with Positive and Negative Syndrome Scale (PANSS) total scores.

\section{The Role of HoFC in Functional Lateralization}

In addition to the functional integration between the two brain hemispheres, another key organization principle of the human brain is functional lateralization. Functional lateralization is acquired by comparing the brain activity patterns between a region in one hemisphere and its contralateral homotopic counterpart in the other (Tomasi and Volkow, 2012; Nielsen et al., 2013). Previous studies have reported that HoFC is related to functional lateralization. Tzourio-Mazoyer et al. (2016) investigated this relationship in 297 healthy volunteers, including 153 left-handers (LHs). They found a significant negative correlation between resting-state HoFC and language task-induced functional lateralization, suggesting a translation from enhanced interhemispheric cooperation in the resting state into increased interhemispheric cooperation during the language production. In addition, all the subjects in this study were classified into three different types according to functional lateralization of language task: typicals, strong-atypicals (only LHs) and ambilaterals. A handedness effect on HoFC was also discovered by the lateralization subgroup comparison, with left-handed ambilaterals having significantly greater HoFC than the other groups. Gracia-Tabuenca et al. (2018) also examined these two brain functional properties, both defined based on rs-FC. Their results identified that HoFC was strongly negatively correlated with functional lateralization across the whole human brain.

Combined with previous findings of the fundamental role of CC, HoFC under resting state might reflect the effect of CC on functional lateralization (Tzourio-Mazoyer et al., 2016). In fact, many studies have demonstrated the excitatory role of CC in interhemispheric processing (Galaburda et al., 1985, 1990). The CC may enforce cerebral processing integration between the left and right hemispheres (Lezak et al., 2004) and activate the unstimulated hemisphere (Yazgan et al., 1995). According to this hypothesis, more symmetric brains have stronger interhemispheric connections, indicating a correlation between a smaller CC and greater lateralization (Bloom and Hynd, 2005). The significant negative correlations between HoFC and functional lateralization in studies above (TzourioMazoyer et al., 2016; Gracia-Tabuenca et al., 2018) therefore 
provided supporting evidence for the excitatory role of the CC in interhemispheric processing in the human brain.

\section{FUTURE CONSIDERATIONS}

We will elaborate several specific aspects about HoFC in the following sections, which deserve particular consideration in future studies.

\section{Lifespan Trajectory of HoFC}

By means of a range of neuroimaging methods, complex changes in the structure and function of the human brain across the lifespan are becoming accessible for analysis (Cao et al., 2014). As early as the fetal period during the second half of gestation, many human brain networks, involving both corticocortical and thalamocortical connections, are initially appeared and established (Kostović and Jovanov-Milošević, 2006). Findings from infants using HoFC under resting state suggested that the developing human brain might increase its interhemispheric functional symmetry to govern multiple functional systems, which might be a general principle in the first year of life (Smyser et al., 2010; Emerson et al., 2016). Therefore, the exploration of functional integration between the two brain hemispheres should also start at earlier stages of brain development such as the fetal or neonatal stage. In most existing lifespan HoFC studies, the age range of subjects were disequilibrium and mostly located at young adults group (Zuo et al., 2010; Tzourio-Mazoyer et al., 2016; Zhao et al., 2020). In future studies, it is warranted to map out lifespan trajectories of HoFC for different homologous regions, using a uniformly distributed lifespan cross-sectional or longitudinal data.

\section{Genetic Basis of HoFC}

Many studies have shown that resting-state FC and networks are considerably heritable, suggesting that HoFC might be influenced by genetic factors (Glahn et al., 2010; Ge et al., 2017). As the structural foundation of HoFC between the two brain hemispheres, the CC is found highly heritable as well. For example, previous studies have confirmed both the size and microstructure of the CC are strongly controlled by genetic factors (Kanchibhotla et al., 2014; Woldehawariat et al., 2014; Vuoksimaa et al., 2017). To date, it remains unexplored whether and how genetic factors influence HoFC. In the future, it would be interesting to investigate the heritability of HoFC at multiple levels by employing advanced imaging genetics techniques, such as twin study with ACE model, genome-wide association study (GWAS) or gene set enrichment analysis (GSEA). The findings would help to understand the relationship between interhemispheric functional integration and genetic factors, as well as environmental factors.

\section{HoFC Under Tasking State}

Compared to rs-fMRI, tasking-state fMRI (ts-fMRI) has been largely used to identify functional activities evoked by specific cognitive tasks. Measurements of brain activity actually reflect a mixture of spontaneous and evoked activities during a specific task. Recently, more and more studies started focusing on tasking-state functional connectivity (ts-FC). Many functional organization properties of the human brain under resting state have been recognized during the tasking state. Across dozens of different tasks, Cole et al. (2014) identified a whole-brain network architecture, which was highly similar to the one presented in resting state. In addition, there was a small but consistent group of changes across different tasks, suggesting the existence of a task-general network architecture distinguished from the resting state. These results indicated that the functional architecture of the human brain under tasking state was likely composed of an intrinsic architecture that appeared under resting state and secondarily shaped by evoked task-general and task-specific changes. In the future, the similarities and differences of HoFC between the resting state and tasking state can be thoroughly explored, which may enhance the understanding about the relationship between the two functional states of the human brain, from an interhemispheric functional integration perspective.

\section{The Functional Meaning of HoFC to Cognition in Healthy People}

To better understand cognitive consequence of functional integration between the two brain hemispheres, more studies are encouraged to explore the functional meaning of HoFC in human brain. As reviewed above, HoFC has been found to be related with particular cognitions, behaviors and specific symptom in both healthy and diseases. In healthy people, studies revealed only a few cognitions related with regional HoFC under resting state, such as executive function, visuospatial attention, language, etc. The association with cognition was more frequently reported in brain disease than healthy people, which might due to the limited individual difference of HoFC in healthy people.

Currently, some available public data, such as the Human Connectome Project (HCP) or UK biobank, provide both neuroimaging and behavior data in a large sample of normal people, allowing for thorough investigation of brain-cognition relationships with a strong statistical power. More studies are encouraged to directly investigate the HoFC-cognition associations in healthy people. By studying these large sample datasets alone or combining with them, researchers may capture subtle associations between the HoFC and complex cognitions/behaviors. Advanced analysis techniques are encouraged for the investigation of HoFC-cognition relationship as well, such as multivariable correlation or machine learning.

\section{CONCLUSION}

This article reviewed existing studies of HoFC under resting state that reflects to some degree functional integration between the two brain hemispheres. Future studies are encouraged to better characterize lifespan trajectories, genetic basis, taskingstate modulation, cognitive correlates of HoFC. 


\section{AUTHOR CONTRIBUTIONS}

$\mathrm{XJ}, \mathrm{XL}$, and GG contributed to the conception and design of the review. XJ and XL wrote the first draft of the manuscript. All authors contributed to manuscript revision and approved it for publication.

\section{REFERENCES}

Biswal, B., Zerrin Yetkin, F., Haughton, V. M., and Hyde, J. S. (1995). Functional connectivity in the motor cortex of resting human brain using echo-planar mri. Magn. Reson. Med. 34, 537-541. doi: 10.1002/mrm.1910340409

Bloom, J. S., and Hynd, G. W. (2005). The role of the corpus callosum in interhemispheric transfer of information: excitation or inhibition? Neuropsychol. Rev. 15, 59-71. doi: 10.1007/s11065-005-6252-y

Cao, M., Wang, J.-H., Dai, Z.-J., Cao, X.-Y., Jiang, L.-L., Fan, F.-M., et al. (2014). Topological organization of the human brain functional connectome across the lifespan. Dev. Cogn. Neurosci. 7, 76-93. doi: 10.1016/j.dcn.2013.11.004

Chen, J., Sun, D., Shi, Y., Jin, W., Wang, Y., Xi, Q., et al. (2020). ). Altered static and dynamic voxel-mirrored homotopic connectivity in subacute stroke patients: a resting-state fMRI study. Brain Imaging Behav. doi: 10.1007/s11682-02000266-x [Epub ahead of print].

Chen, Q., Beaty, R. E., Cui, Z., Sun, J., He, H., Zhuang, K., et al. (2019). Brain hemispheric involvement in visuospatial and verbal divergent thinking. Neuroimage 202:116065. doi: 10.1016/j.neuroimage.2019. 116065

Cole, M. W., Bassett, D. S., Power, J. D., Braver, T. S., and Petersen, S. E. (2014). Intrinsic and task-evoked network architectures of the human brain. Neuron 83, 238-251. doi: 10.1016/j.neuron.2014.05.014

Cordes, D., Haughton, V. M., Arfanakis, K., Wendt, G. J., Turski, P. A., Moritz, C. H., et al. (2000). Mapping functionally related regions of brain with functional connectivity MR imaging. Am. J. Neuroradiol. 21, 1636-1644.

Duffy, F. H., McAnulty, G. B., and Albert, M. S. (1996). Effects of age upon interhemispheric EEG coherence in normal adults. Neurobiol. Aging 17, 587599. doi: 10.1016/0197-4580(96)00007-3

Emerson, R. W., Gao, W., and Lin, W. (2016). Longitudinal study of the emerging functional connectivity asymmetry of primary language regions during infancy. J. Neurosci. 36, 10883-10892. doi: 10.1523/JNEUROSCI.3980-15.2016

Fan, H., Yang, X., Zhang, J., Chen, Y., Li, T., and Ma, X. (2018). Analysis of voxelmirrored homotopic connectivity in medication-free, current major depressive disorder. J. Affect. Disord. 240, 171-176. doi: 10.1016/j.jad.2018.07.037

Fox, M. D., and Raichle, M. E. (2007). Spontaneous fluctuations in brain activity observed with functional magnetic resonance imaging. Nat. Rev. Neurosci. 8, 700-711. doi: 10.1038/nrn2201

Friston, K. (2004). Functional Integration in the Brain in Human Brain Function. Amsterdam: Elsevier, 971-997. doi: 10.1016/B978-012264841-0/50050-0

Galaburda, A. M., Rosen, G. D., and Sherman, G. F. (1990). Individual variability in cortical organization: Its relationship to brain laterality and implications to function. Neuropsychologia 28, 529-546. doi: 10.1016/0028-3932(90)90032-J

Galaburda, A. M., Sherman, G. F., Rosen, G. D., Aboitiz, F., and Geschwind, N. (1985). Developmental dyslexia: four consecutive patients with cortical anomalies. Ann. Neurol. 18, 222-233. doi: 10.1002/ana.410180210

Ge, T., Holmes, A. J., Buckner, R. L., Smoller, J. W., and Sabuncu, M. R. (2017). Heritability analysis with repeat measurements and its application to restingstate functional connectivity. Proc. Natl. Acad. Sci. U.S.A. 114, 5521-5526. doi: $10.1073 /$ pnas. 1700765114

Gee, D. G., Biswal, B. B., Kelly, C., Stark, D. E., Margulies, D. S., Shehzad, Z., et al. (2011). Low frequency fluctuations reveal integrated and segregated processing among the cerebral hemispheres. Neuroimage 54, 517-527. doi: 10.1016/j.neuroimage.2010.05.073

Glahn, D. C., Winkler, A. M., Kochunov, P., Almasy, L., Duggirala, R., Carless, M. A., et al. (2010). Genetic control over the resting brain. Proc. Natl. Acad. Sci.U.S.A. 107, 1223-1228. doi: 10.1073/pnas.0909969107

Gotts, S. J., Jo, H. J., Wallace, G. L., Saad, Z. S., Cox, R. W., and Martin, A. (2013). Two distinct forms of functional lateralization in the human brain. Proc. Natl. Acad. Sci. U.S.A. 110, E3435-E3444. doi: 10.1073/pnas.1302581110

\section{FUNDING}

This work was supported by the National Science Foundation of China (grant numbers 81671772 and 91732101 ), the Research Fund of PLA of China (grant number AWS17J011), and the Fundamental Research Funds for the Central Universities.

Gracia-Tabuenca, Z., Moreno, M. B., Barrios, F. A., and Alcauter, S. (2018). Hemispheric asymmetry and homotopy of resting state functional connectivity correlate with visuospatial abilities in school-age children. Neuroimage 174, 441-448. doi: 10.1016/j.neuroimage.2018.03.051

Guo, W., Cui, X., Liu, F., Chen, J., Xie, G., Wu, R., et al. (2018). Decreased interhemispheric coordination in the posterior default-mode network and visual regions as trait alterations in first-episode, drug-naive major depressive disorder. Brain Imaging Behav. 12, 1251-1258. doi: 10.1007/s11682-0179794-8

Guo, W., Liu, F., Dai, Y., Jiang, M., Zhang, J., Yu, L., et al. (2013). Decreased interhemispheric resting-state functional connectivity in first-episode, drugnaive major depressive disorder. Prog. Neuro-Psychopharmacol. Biol. Psychiatry 41, 24-29. doi: 10.1016/j.pnpbp.2012.11.003

Hermesdorf, M., Sundermann, B., Feder, S., Schwindt, W., Minnerup, J., Arolt, V., et al. (2016). Major depressive disorder: findings of reduced homotopic connectivity and investigation of underlying structural mechanisms. Hum. Brain Mapp. 37, 1209-1217. doi: 10.1002/hbm.23097

Honey, C. J., Sporns, O., Cammoun, L., Gigandet, X., Thiran, J. P., Meuli, R., et al. (2009). Predicting human resting-state functional connectivity from structural connectivity. Proc. Natl. Acad. Sci. U.S.A. 106, 2035-2040. doi: 10.1073/pnas. 0811168106

Hoptman, M. J., Zuo, X.-N., D’Angelo, D., Mauro, C. J., Butler, P. D., Milham, M. P., et al. (2012). Decreased interhemispheric coordination in schizophrenia: a resting state fMRI study. Schizophr. Res. 141, 1-7. doi: 10.1016/j.schres.2012. 07.027

Horwitz, B., Duara, R., and Rapoport, S. I. (1984). Intercorrelations of glucose metabolic ates between brain regions: application to healthy males in a state of reduced sensory input. J. Cereb. Blood Flow Metab. 4, 484-499. doi: 10.1038/ jcbfm.1984.73

Innocenti, G. M. (1986). Postnatal development of corticocortical connections. Ital. J. Neurol. Sci. 9, 600-613. doi: 10.1523/jneurosci.09-02-00600.1989

Jo, H. J., Saad, Z. S., Gotts, S. J., Martin, A., and Cox, R. W. (2012). Quantifying agreement between anatomical and functional interhemispheric correspondences in the resting brain. PLoS One 7:e48847. doi: 10.1371/journal. pone. 0048847

Johnston, J. M., Vaishnavi, S. N., Smyth, M. D., Zhang, D., He, B. J., Zempel, J. M., et al. (2008). Loss of resting interhemispheric functional connectivity after complete section of the corpus callosum. J. Neurosci. 28, 6453-6458. doi: 10.1523/JNEUROSCI.0573-08.2008

Joliot, M., Jobard, G., Naveau, M., Delcroix, N., Petit, L., Zago, L., et al. (2015). AICHA: an atlas of intrinsic connectivity of homotopic areas. J. Neurosci. Methods 254, 46-59. doi: 10.1016/j.jneumeth.2015.07.013

Kanchibhotla, S. C., Mather, K. A., Thalamuthu, A., Zhuang, L., Schofield, P. R., Kwok, J. B. J., et al. (2014). Genetics of microstructure of the corpus callosum in older adults. PLoS One 9:e113181. doi: 10.1371/journal.pone.011 3181

Kelly, C., Zuo, X.-N., Gotimer, K., Cox, C. L., Lynch, L., Brock, D., et al. (2011). Reduced interhemispheric resting state functional connectivity in cocaine addiction. Biol. Psychiatry 69, 684-692. doi: 10.1016/j.biopsych.2010. 11.022

Kostović, I., and Jovanov-Milošević, N. (2006). The development of cerebral connections during the first 20-45 weeks' gestation. Semin. Fetal Neonatal. Med. 11, 415-422. doi: 10.1016/j.siny.2006.07.001

Lezak, M. D., Howieson, D. B., Loring, D. W., Hannay, J. H., and Fischer, J. S. (2004). Neuropsychological Assessment. New York, NY: Oxford University Press.

Lowe, M. J., Mock, B. J., and Sorenson, J. A. (1998). Functional connectivity in single and multislice echoplanar maging using restingstate fluctuations. Neuroimage 7, 119-132. doi: 10.1006/nimg.1997. 0315 
Luo, C., Guo, X., Song, W., Zhao, B., Cao, B., Yang, J., et al. (2015). Decreased resting-state interhemispheric functional connectivity in Parkinson's disease. Biomed. Res. Int. 2015, 1-8. doi: 10.1155/2015/69 2684

Luo, X., Li, K., Zeng, Q., Huang, P., Jiaerken, Y., Qiu, T., et al. (2018). Decreased bilateral FDG-PET uptake and inter-hemispheric connectivity in multi-domain amnestic mild cognitive impairment patients: a preliminary study. Front. Aging Neurosci. 10:161. doi: 10.3389/fnagi.2018.00161

Mollink, J., Smith, S. M., Elliott, L. T., Kleinnijenhuis, M., Hiemstra, M., AlfaroAlmagro, F., et al. (2019). The spatial correspondence and genetic influence of interhemispheric connectivity with white matter microstructure. Nat. Neurosci. 22, 809-819. doi: 10.1038/s41593-019-0379-2

Nielsen, J. A., Zielinski, B. A., Ferguson, M. A., Lainhart, J. E., and Anderson, J. S. (2013). An evaluation of the left-brain vs. right-brain hypothesis with resting state functional connectivity magnetic resonance imaging. PLoS One 8:e71275. doi: 10.1371/journal.pone.0071275

Nuttall, H. E., Kennedy-Higgins, D., Devlin, J. T., and Adank, P. (2018). Modulation of intra- and inter-hemispheric connectivity between primary and premotor cortex during speech perception. Brain Lang. 187, 74-82. doi: 10. 1016/j.bandl.2017.12.002

Owen, J. P., Li, Y.-O., Yang, F. G., Shetty, C., Bukshpun, P., Vora, S., et al. (2013). Resting-State Networks and the Functional Connectome of the Human Brain in Agenesis of the Corpus Callosum. Brain Connect. 3, 547-562. doi: 10.1089/ brain.2013.0175

Quigley, M., Cordes, D., Turski, P., Moritz, C., Haughton, V., Seth, R., et al. (2003). Role of the corpus callosum in functional connectivity. AJNR Am. J. Neuroradiol. 24, 208-212.

Ren, J., Lei, D., Yang, T., An, D., Xiao, F., Li, L., et al. (2015). Increased interhemispheric resting-state functional connectivity in paroxysmal kinesigenic dyskinesia: a resting-state fMRI study. J. Neurol. Sci. 351, 93-98. doi: 10.1016/j.jns.2015.02.046

Roland, J. L., Snyder, A. Z., Hacker, C. D., Mitra, A., Shimony, J. S., Limbrick, D. D. et al. (2017). On the role of the corpus callosum in interhemispheric functional connectivity in humans. Proc. Natl. Acad. Sci. U.S.A. 114, 13278-13283. doi: 10.1073/pnas.1707050114

Salvador, R., Suckling, J., Coleman, M. R., Pickard, J. D., Menon, D., and Bullmore, E. (2005). Neurophysiological architecture of functional magnetic resonance images of human brain. Cereb. Cortex 15, 1332-1342. doi: 10.1093/cercor/ bhi016

Shen, K., Mišić, B., Cipollini, B. N., Bezgin, G., Buschkuehl, M., Hutchison, R. M., et al. (2015). Stable long-range interhemispheric coordination is supported by direct anatomical projections. Proc. Natl. Acad. Sci. U.S.A. 112, 6473-6478. doi: 10.1073/pnas.1503436112

Smyser, C. D., Inder, T. E., Shimony, J. S., Hill, J. E., Degnan, A. J., Snyder, A. Z., et al. (2010). Longitudinal analysis of neural network development in preterm infants. Cereb. Cortex 20, 2852-2862. doi: 10.1093/cercor/bhq035

Stark, D. E., Margulies, D. S., Shehzad, Z. E., Reiss, P., Kelly, A. M. C., Uddin, L. Q., et al. (2008). Regional variation in interhemispheric coordination of intrinsic hemodynamic fluctuations. J. Neurosci. 28, 13754-13764. doi: 10.1523/ JNEUROSCI.4544-08.2008

Tobyne, S. M., Boratyn, D., Johnson, J. A., Greve, D. N., Mainero, C., and Klawiter, E. C. (2016). A surface-based technique for mapping homotopic interhemispheric connectivity: development, characterization, and clinical application. Hum. Brain Mapp. 37, 2849-2868. doi: 10.1002/hbm.23214

Tomasi, D., and Volkow, N. D. (2012). Laterality Patterns of Brain Functional Connectivity: Gender Effects. Cereb. Cortex 22, 1455-1462. doi: 10.1093/cercor/ bhr230
Tononi, G., Sporns, O., and Edelman, G. M. (1994). A measure for brain complexity: relating functional segregation and integration in the nervous system. Proc. Natl. Acad. Sci. U.S.A. 91, 5033-5037. doi: 10.1073/pnas.91.11. 5033

Tzourio-Mazoyer, N., Joliot, M., Marie, D., and Mazoyer, B. (2016). Variation in homotopic areas' activity and inter-hemispheric intrinsic connectivity with type of language lateralization: an FMRI study of covert sentence generation in 297 healthy volunteers. Brain Struct. Funct. 221, 2735-2753. doi: 10.1007/s00429015-1068-x

Tzourio-Mazoyer, N., Landeau, B., Papathanassiou, D., Crivello, F., Etard, O., Delcroix, N., et al. (2002). Automated anatomical labeling of activations in SPM using a macroscopic anatomical parcellation of the MNI MRI single-subject brain. Neuroimage 15, 273-289. doi: 10.1006/nimg.2001. 0978

van den Heuvel, M. P., and Sporns, O. (2013). An anatomical substrate for integration among functional networks in human cortex. J. Neurosci. 33, 1448914500. doi: 10.1523/JNEUROSCI.2128-13.2013

Vuoksimaa, E., Panizzon, M. S., Hagler, D. J. Jr, Hatton, S. N., Fennema-Notestine, C., Rinker, D., et al. (2017). Heritability of white matter microstructure in late middle age: a twin study of tract-based fractional anisotropy and absolute diffusivity indices. Hum. Brain Mapp. 38, 2026-2036. doi: 10.1002/hbm. 23502

Woldehawariat, G., Martinez, P. E., Hauser, P., Hoover, D. M., Drevets, W. W. C., and McMahon, F. J. (2014). Corpus callosum size is highly heritable in humans, and may reflect distinct genetic influences on ventral and rostral regions. PLoS One 9:e99980. doi: 10.1371/journal.pone.0099980

Yang, T., Ren, J., Li, Q., Li, L., Lei, D., Gong, Q., et al. (2014). Increased interhemispheric resting-state in idiopathic generalized epilepsy with generalized tonic-clonic seizures: a resting-state fMRI study. Epilepsy Res. 108, 1299-1305. doi: 10.1016/j.eplepsyres.2014.06.010

Yazgan, M. Y., Wexler, B. E., Kinsbourne, M., Peterson, B., and Leckman, J. F. (1995). Functional significance of individual variations in callosal area. Neuropsychologia 33, 769-779. doi: 10.1016/0028-3932(95)00 018-X

Ye, M., Guo, Z., Li, Z., Lin, X., Li, J., Jiang, G., et al. (2020). Aberrant interhemispheric coordination characterizes the progression of minimal hepatic encephalopathy in patients with HBV-related cirrhosis. NeuroImage Clin. 25:102175. doi: 10.1016/j.nicl.2020.102175

Zhao, J., Manza, P., Wiers, C., Song, H., Zhuang, P., Gu, J., et al. (2020). Agerelated decreases in interhemispheric resting-state functional connectivity and their relationship with executive function. Front. Aging Neurosci 12:20. doi: 10.3389/fnagi.2020.00020

Zuo, X.-N., Kelly, C., Di Martino, A., Mennes, M., Margulies, D. S., Bangaru, S., et al. (2010). Growing together and growing apart: regional and sex differences in the lifespan developmental trajectories of functional homotopy. J. Neurosci. 30, 15034-15043. doi: 10.1523/JNEUROSCI.2612-10.2010

Conflict of Interest: The authors declare that the research was conducted in the absence of any commercial or financial relationships that could be construed as a potential conflict of interest.

Copyright (c) 2020 Jin, Liang and Gong. This is an open-access article distributed under the terms of the Creative Commons Attribution License (CC BY). The use, distribution or reproduction in other forums is permitted, provided the original author(s) and the copyright owner(s) are credited and that the original publication in this journal is cited, in accordance with accepted academic practice. No use, distribution or reproduction is permitted which does not comply with these terms. 